\title{
[gw22-e0183] STUDY OF THE MECHANISIMS OF PROTECTIVE EFFECT OF DIAZOXIDE ON THE MYOCARDIAL MITOCHONDRIAL FUNCTIONS AFTER ASPHYXIA IN NEONATAL RATS
}

Fang Xiaoyi, Li Yuguang, Lin Niyang, Chen Yuxia

First Affiliated Hospital of Shantou University Medical College

\subsection{6/heartjnl-2011-300867.98}

Objectives To explore the mechanisms of protective effects of diazoxide, a mitoK $\mathrm{K}_{\text {ATP }}$ opener (KCO), on mitochondrial functions through detection of the opening of mitochondrial permeability transition pore (MPTP) in hypoxic ischemic myocardial damage model induced by asphyxia in neonatal rats.

Methods Caesarean sections were undertaken in female $\mathrm{SD}$ rats on the 21 st day after pregnancy. The uterine arteries were clamped for $30 \mathrm{~min}$ followed by releasing for $1 \mathrm{~h}$ and the pups were divided randomly into asphyxia, diazoxide (Dia), Glibenclamide (Gli), and solvent $(\mathrm{NaOH})$ group. The uterine arteries were isolated but not clamped in control group. diazoxide was injected intraperitoneally (intraperitoneal) in $3 \mathrm{mg} / \mathrm{kg}$ to the rats in Dia group. diazoxide $(3 \mathrm{mg} / \mathrm{kg})$ and Glibenclamide $(300 \mu \mathrm{g} / \mathrm{kg})$ were injected intraperitoneal to the rats in Gli group. $0.5 \mathrm{ml} \mathrm{NaOH}(0.1 \mathrm{mM})$ was injected intraperitoneal to the rats in solvent group. All the neonatal rats were sacrificed $24 \mathrm{~h}$ after birth. Serum cardiac troponin I (cTnI) levels were detected by ELISA. Myocardial ischemic areas were detected by TTC staining. Cardiac apoptosis inducing factors (AIF) were detected by immunohistochemical staining. The opening degree of MPTP was detected by fluorospectrophotometry.

Results Serum cTnI levels were $0.08 \pm 0.04,0.40 \pm 0.29$, $0.10 \pm 0.04,0.33 \pm 0.17$ and $0.34 \pm 0.20(\mu \mathrm{g} / \mathrm{l})(\mathrm{p}<0.01)$, the myocardial ischemic areas were $8.01 \pm 3.48,42.50 \pm 15.90,14.79 \pm 3.98$, $31.51 \pm 20.86$ and $28.37 \pm 14.36(\%)(p<0.01)$, the AIF-staining positive cells in myocardial tissue slices were $9.70 \pm 3.06$, $77.50 \pm 11.81,44.60 \pm 17.49,70.70 \pm 14.36$ and $73.60 \pm 15.19(\%)$ $(p<0.01)$ and the opening degrees of MPTP were 118.10 \pm 19.10 , $79.40 \pm 10.57,106.40 \pm 14.61,72.50 \pm 11.21$ and $76.20 \pm 3.79$ (RFU) $(\mathrm{p}<0.01)$ in control, asphyxia, diazoxide, Glibenclamide and solvent group, respectively. The serum levels of cTnI, the myocardial ischemic areas, the AIF expressions and the opening degrees of MPTP were significantly increased in asphyxia group compared with control group. The serum level of cTnI and expression value of AIF was positively correlated with the opening degree of MPTP. After treatment of diazoxide, cTnI levels, the myocardial ischemic areas, the AIF expressions and the opening degrees of MPTP were decreased.

Conclusions There are myocardial injuries in neonatal rats after asphyxia. Opening of myocardial MPTP, which may induce releasing of AIF, are the main causes of myocardial injury. After treatment with diazoxide, the myocardial injuries are improved. The protective effects of diazoxide on the myocardial mitochondria include inhibition of opening of MPTP. 